\section{POS0678 DOES SILDENAFIL IMPROVE ENDOTHELIAL DYSFUNCTION IN RHEUMATOID ARTHRITIS? - A PILOT CLINICAL TRIAL}

K. Liang ${ }^{1}$, D. Landsittel ${ }^{2}$, Y. Li ${ }^{2}$, L. Hope ${ }^{3}$, L. Ruffalo ${ }^{3}$, J. Peat-Fircak ${ }^{3}$, J. Avolio ${ }^{4}$, P. Biswas ${ }^{3}$, E. Roth ${ }^{3}$, M. Simon ${ }^{5}$, L. Moreland ${ }^{6} .{ }^{1}$ University of Pittsburgh, Rheumatology and Clinical Immunology, Pittsburgh, PA, United States of America; ${ }^{2}$ University of Pittsburgh, Biomedical Informatics, Pittsburgh, United States of America; ${ }^{3}$ University of Pittsburgh, Rheumatology and Clinical Immunology, Pittsburgh, United States of America; ${ }^{4}$ University of Pittsburgh, Clinical and Translational Science Institute, Pittsburgh, PA, United States of America; ${ }^{5}$ University of California San Francisco, Cardiology, San Francisco, CA, United States of America; ${ }^{6}$ University of Colorado, Rheumatology, Aurora, $\mathrm{CO}$, United States of America

Background: Rheumatoid arthritis (RA) is independently associated with an increased risk of cardiovascular disease (CVD). One of the early stages of atherosclerosis is endothelial dysfunction, which is increased in RA. Using drugs to target endothelial dysfunction is a promising novel strategy for CVD prevention in RA. Sildenafil has been shown to improve endothelial function in diabetics, who have similar increased CVD risk. Our hypothesis was that sildenafil use may be a novel primary CVD prevention strategy in RA.

Objectives: To determine if sildenafil use in RA patients improves endothelia dysfunction (as measured by brachial artery flow-mediated dilation [FMD] and peripheral arterial tone [PAT]), as well as serum inflammatory and atherosclerosis biomarkers.

Methods: This NIH-funded study was a phase II, randomized double-blind placebo-controlled crossover efficacy trial of 25 RA patients, with no known history of CVD, but at least one traditional CVD risk factor. Patients were randomized 1:1 to receive either sildenafil or placebo for 3 months, then after a 2-week washout, crossed over to each respective group for an additional 3 months. Vascular studies (FMD and PAT) and serum atherosclerosis biomarkers (e-Selectin, ICAM-1, VCAM-1) were performed at baseline, 3 months pre- and post-washout, and 6 months. Adverse events were collected. Given the cross-over design, analyses included a random effects model for within-subject comparisons of sildenafil versus placebo periods, adjusting for the baseline (FMD or EndoPAT) within that period and a term for treatment order. All tests were 2-sided with $\alpha=0.05$.

Results: A total of 233 subjects were assessed for eligibility, with 25 subjects being randomized after written informed consent. $A$ total of 13 subjects were randomized to placebo first, and 12 to sildenafil first. Baseline characteristics were similar between those randomized to Placebo vs. Sildenafil first. Mean age was $62.0+/-10.9$ years $84 \%$ were female; and $92 \%$ were white. A total of 6 adverse events experienced in 3 subjects occurred. The primary endpoint (increase in \%FMD in Sildenafil period vs. Placebo period) was not significant ( $p=0.19)$. However, note the study was powered at $80 \%$ to detect an effect size of 0.37 for change in \%FMD or biomarker with a sample size of 60 , not 25 . However, sildenafil use was associated with a significant increase (improvement) by 0.200 units of PAT ratio $(\mathrm{p}=0.003)$ compared with placebo, adjusted by treatment order and baseline PAT ratio (within the given treatment period). Exploratory linear mixed models comparing e-Selectin, ICAM-1, and VCAM-1 between Sildenafil vs. Placebo periods, adjusted for treatment order and the baseline biomarker level, did not show any significant differences except for ICAM-1 (55.3 units higher in Sildenafil vs. Placebo periods, $\mathrm{p}=0.011$ ).

Conclusion: In this pilot trial of 25 RA subjects, sildenafil use was associated with a significant increase (improvement) in endothelial function as measured by PAT. However, there was no significant difference in FMD. The study is limited due to the small sample size, which was impacted by slow recruitment as well as the COVID-19 pandemic. Future larger studies are required to assess whether other PDE5 inhibitors may improve endothelial dysfunction in RA and other autoimmune disease patients at high risk of CVD.

REFERENCES:

[1] Maradit-Kremers H, Crowson CS, Nicola PJ, et al. Increased unrecognized coronary heart disease and sudden deaths in rheumatoid arthritis: a population-based cohort study. Arthritis Rheum 2005;52:402-11.

[2] Peters MJ, van Halm VP, Voskuyl AE, et al. Does rheumatoid arthritis equal diabetes mellitus as an independent risk factor for cardiovascular disease? A prospective study. Arthritis Rheum 2009;61:1571-9.

[3] Deyoung L, Chung E, Kovac JR, et al. Daily use of sildenafil improves endothelial function in men with type 2 diabetes. J Andrology 2012;33:176-80.

Disclosure of Interests: None declared

DOI: 10.1136/annrheumdis-2021-eular.4069

\section{POS0679 \\ A NEW QUESTIONNAIRE AND SCORE (MISA) FOR ASSESSING METHOTREXATE INTOLERANCE IN RHEUMATOID ARTHRITIS}

V. Dhir ${ }^{1}$, D. V ${ }^{1}$, S. Jain ${ }^{1}$, V. Pai ${ }^{2}$, A. Sharma ${ }^{1}$, S. K. Sharma ${ }^{1}$, G. Naidu' ${ }^{1}$ S. Jain ${ }^{1}$. ${ }^{1}$ Postgraduate Institute of Medical Education and Research, Rheumatology Division, Department of Internal Medicine, Chandigarh, India; ${ }^{2}$ All India Institute of Medical Sciences, Rishikesh, Rheumatology, Medicine, Rishikesh, India
Background: Methotrexate (MTX) intolerance refers to unpleasant symptoms that accompany use of MTX and may lead to its discontinuation. However, it lacks a validated score in RA patients; with the only option being to use the MISS score which was validated for use in children and not adults.

Objectives: To develop and validate a questionnaire and score (s) for measuring MTX intolerance and its severity in rheumatoid arthritis.

Methods: A 10-item questionnaire called 'Methotrexate Intolerance and Severity assessment in Adults' (MISA) was validated in 105 RA patients. A score was calculated by adding the scores of first 7 questions ( 0 to 3 based on severity on symptoms), to last 3 questions ( 0 or 1 ); it ranged from 0 to 24 (MISA score) and was assessed for correctly classifying MTX intolerance (compared to an interview) by ROC analysis. Its area-under-curve (AUC) was compared with 'Methotrexate Intolerance Severity Score' (MISS), developed for children. Subsequently, it was administered to 414 RA patients to assess the prevalence and associations of MTX intolerance. In addition, the MISA cross-products score, that was calculated by adding the cross-products (severity ( 1 to 3 ) $x$ duration per week ( 0.5 to 7 days)) o symptoms, was compared to MISA and MISS for assessing severity of intolerance. Results: In the initial phase, 105 RA patients on MTX $\geq 6$ months were included, a majority were female (87\%), mean age was 51 (13.4) years and methotrexate dose was $18.8 \pm 6 \mathrm{mg} /$ week. Thirty-five ( $33 \%$ ) were found to be intolerant to MTX based on interview. MISA score had a good predictive ability (AUC of 0.904 ), to correctly classify MTX intolerance, and was better than MISS score (AUC of 0.823) (Figure 1A). The optimal cut-off for MISA was $\geq 1$, with a sensitivity and specificity of $91.4 \%$ and $84.3 \%$. Using the MISA score ( $\geq 1), 38.4 \%$ of 414 RA patients were found to have MTX intolerance: with nausea, lethargy and irritability being common symptoms. (Figure 1B, C) On multivariable analysis, age (OR 0.972) and BMI (OR 1.061) were significant predictors of MTX intolerance. (Table 1) On assessing for severity of intolerance, MISA cross product score performed the best, with an area-under-curve of 0.899 (95\% Cl 0.831-0.966), being higher than AUC for MISS and MISA score which were $0.847(95 \% \mathrm{Cl} 0.768-0.927)$ and 0.837 (95\% Cl 0.754-0.920).

Conclusion: MISA is the first validated questionnaire for assessing methotrexate intolerance in rheumatoid arthritis, with the MISA score having a good accuracy (at cut-off $\geq 1$ ), to detect MTX intolerance. Methotrexate intolerance was present in more than one-third of RA patients, with nausea, lethargy and irritability being most common.

Table 1. Baseline characteristics of 414 RA patients.

\begin{tabular}{|c|c|c|c|c|c|}
\hline Variable & $\begin{array}{l}\text { All } \\
(n=414)\end{array}$ & $\begin{array}{l}\text { Tolerant } \\
(n=255)\end{array}$ & $\begin{array}{l}\text { Intolerant } \\
(\mathrm{n}=159)\end{array}$ & $\begin{array}{l}\mathrm{p} \text {-value } \\
\text { tol. vs ntol. }\end{array}$ & $\begin{array}{l}\text { P-value } \\
\text { multivariable } \\
\text { model }\end{array}$ \\
\hline males, $n(\%)$ & $370(89)$ & $231(9$ & $139(87)$ & 0.31 & \\
\hline Age, yrs, mean (SD) & $50(12.5)$ & $51.2(12.6)$ & $48.2(12.2)$ & $0.016^{*}$ & $0.008^{\star \star}$ \\
\hline Duration of RA, yrs, $m$ & $10.0(7.0)$ & $10.8(7.4)$ & $9.6(6.3)$ & 0.168 & \\
\hline $\mathrm{BMI}, \mathrm{Kg} / \mathrm{m}^{2 \mathrm{a}}$, mean (SD) & $24.0(4.9)$ & $23.6(4.9)$ & $24.6(4.7)$ & 0.107 & $0.03^{*}$ \\
\hline RF positive ${ }^{b}, n(\%)$ & $300(73)$ & $191(82)$ & $109(78)$ & 0.29 & \\
\hline CDAl, mean $( \pm \mathrm{SD})$ & $14.0(11.8)$ & $14(12.1)$ & $14.1(11.4)$ & 0.69 & \\
\hline Dose of MTX, mg/wk, mean (SD) & $18.6(5.6)$ & $18.6(5.5)$ & $18.7(5.8)$ & 0.83 & \\
\hline Injectable MTX, n (\%) & $47(11)$ & $25(10)$ & $22(14)$ & 0.21 & \\
\hline Use of FA, $n(\%)$ & $395(95)$ & $241(95)$ & $154(97)$ & 0.27 & \\
\hline her DMARD, $\mathrm{n}$ & $272(66)$ & $160(62)$ & $112(70)$ & 0.11 & \\
\hline $\mathrm{HCQ} n(\%)$ & $209(51)$ & $123(48)$ & $86(54)$ & 0.25 & \\
\hline Predniso & $156(38)$ & $87(34)$ & $69(43)$ & 0.058 & 0.21 \\
\hline Using antiemetics, $\mathrm{n}(\%)$ & $12(3)$ & $1(0.5)$ & $11(7)$ & $<0.001$ & \\
\hline
\end{tabular}

${ }^{a}$ Available for 262 patients ${ }^{\mathrm{b}}$ Available for 372 patients

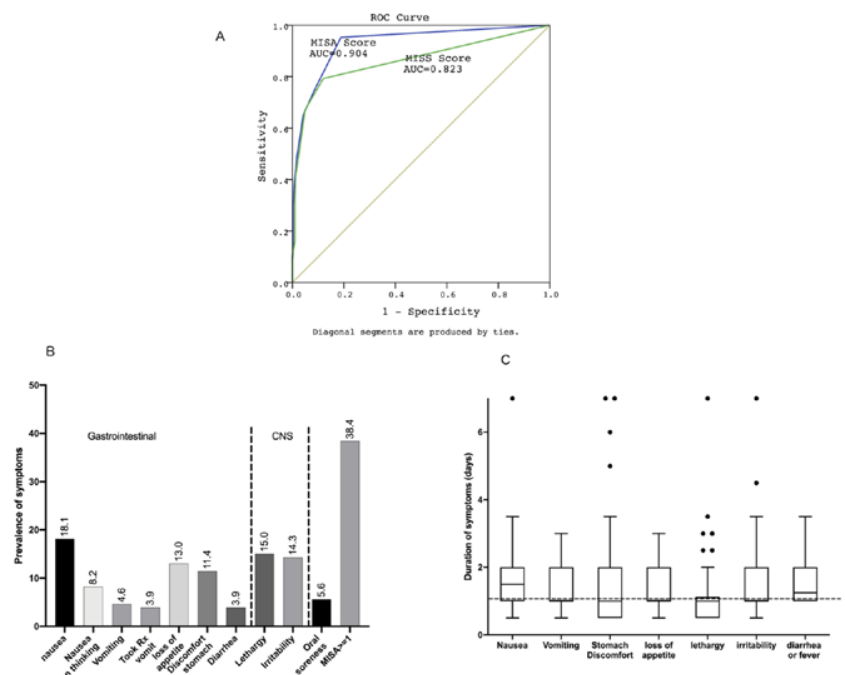


Figure 1. Figure showing the ROC curve for MISA and MISS questionnaires for MTX intolerance (A), Bar diagram showing the prevalence of various symptoms of intolerance in 414 RA patients (B), and Box-and-whiskers plot showing the duration of unpleasant symptoms (C).

Disclosure of Interests: None declared

DOI: 10.1136/annrheumdis-2021-eular.4241

\section{SLE, Sjögren's and APS - treatment_}

\section{POS0680 BELIMUMAB ADD-ON THERAPY MOBILISES MEMORY B CELLS INTO THE CIRCULATION OF PATIENTS WITH SLE}

E. J. Arends ${ }^{1}$, M. Zlei ${ }^{2}$, C. M. Tipton ${ }^{3}$, Z. Osmani ${ }^{1}$, S. Kamerling ${ }^{1}$, T. Rabelink ${ }^{1}$, I. Sanz ${ }^{3}$, J. J. M. Van Dongen ${ }^{2}$, C. Van Kooten ${ }^{1}$, Y. K. O. Teng ${ }^{1} .{ }^{1}$ Leiden University Medical Center (LUMC), Nephrology, Leiden, Netherlands; ${ }^{2}$ Leiden University Medical Center (LUMC), Immunology, Leiden, Netherlands; ${ }^{3}$ Emory University-School of Medicine, Division of Rheumatology, Department of Medicine, Atlanta, United States of America

Background: Belimumab (BLM), a recombinant human monoclonal antibody directed against B-cell activating factor (BAFF), is the first approved biological agent for patients with active severe systemic lupus erythematosus (SLE) and lupus nephritis $(\mathrm{LN})$. There is clinical evidence that combining BLM with B cell depleting therapy can ameliorate disease activity in severe, refractory SLE patients ${ }^{1}$. Although BLM is a B cell directed therapy and has been shown to significantly decrease total $\mathrm{B}$ cells, flow cytometry observations suggest a rapid increase of circulating memory $B$ cells $(\mathrm{MBC})^{2}$.

Objectives: To investigate dynamics of B-cell subsets in SLE patients treated with or without BLM, with a focus on assessing MBC characteristics.

Methods: Extensive B cell subset phenotyping was performed by high-sensitivity (HS) flow cytometry (acquisition of $10^{7}$ leukocytes; per EuroFlow protocols ${ }^{3}$ ) on samples from active LN or SLE patients with major organ involvement treated with standard of care $(\mathrm{SOC})$ consisting of high dose steroids and mycophenolate mofetil combined with or without the addition of BLM. MBC gene expression profiles were characterized with single-cell RNA and $V(D) J$ sequencing (ScRNA-SEQ).

Results: By employing HS flowcytometry, we established that the absolute increase in circulating MBC in SLE and LN patients was significant for patients who initiated BLM (Figure 1). The increase was observed in a broad range of MBC subsets (Unswitched, IgG1+, IgG2+, IgA1+, IgA2+) at 2 and 4 weeks following initiation of BLM treatment. This rise in MBC could hypothetically be attributed to either proliferation of blood MBC, BLM induced migration of tissue-resident MBCs or BLM related retention of tissue-destined MBC in the blood. ScRNA-SEQ analysis of cell cycle gene-expression was performed and established in both groups a non-proliferating phenotype [in approximately 94\%] of MBC post-treatment, including absence of MKI67 as active proliferation marker. Clonal diversity analysis comparing week 2 with baseline revealed an unexpected decrease of the largest MBC clones in BLM, whereas no change in clonality was observed with $\mathrm{SOC}$ alone. Together these data indicate that proliferation is unlikely to be responsible for the observed increase in MBC by BLM. Furthermore, a clear difference was found in gene-expression levels between both treatment groups:
SOC

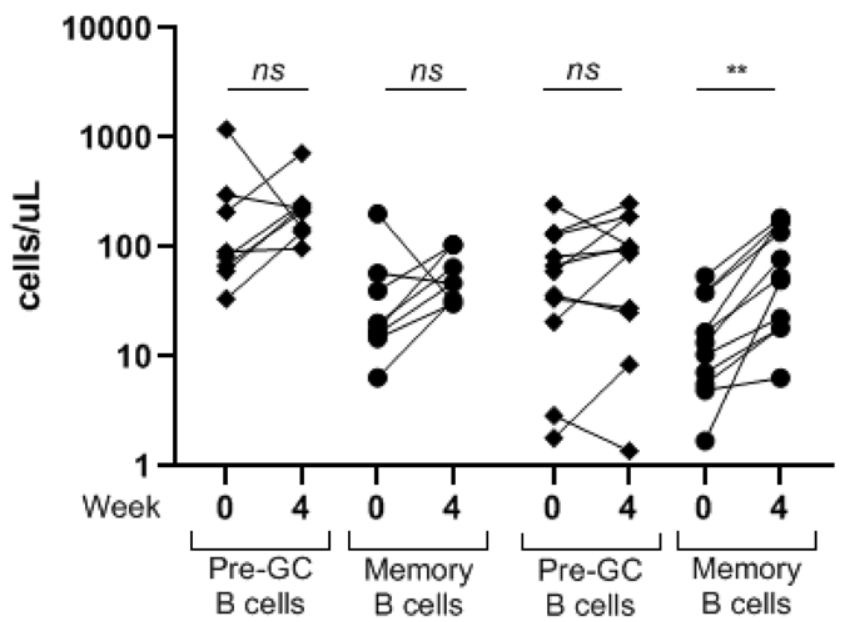

Figure 1. Change in pre-germinal center and memory B cell counts from baseline to week 4 of patients with SLE or LN treated with SOC $(n=8)$ or SOC+BLM $(n=11)$
BLM was responsible for the upregulation of 72 vs 10 genes in SOC, likewise 162 vs 32 genes were downregulated. Most importantly, a significant downregulation of the migration genes SELL (CD62L), CCR7, ITGB1, RAC2 and ICAM2, were specifically seen in BLM treated patients. This may reflect disrupted lymphocyte trafficking, preventing MBCs from transmigrating from the blood into tissue owing to reduced migration molecules, or preventing MBCs from being retained at the tissue level owing to reduction in tissue adhesion proteins.

Conclusion: The addition of BLM to SOC significantly increases MBCs in patients with SLE independently of proliferation, accompanied by a strong mod ulation of gene expression, including reduced expression of migration markers pointing towards disrupted lymphocyte trafficking. These data may have important implications for improving treatment strategies in patients with LN or severe SLE, as a deeper depletion of autoreactive MBCs could be established by adding B-cell-depleting therapy after the initiation of BLM.

\section{REFERENCES:}

[1] Arends EJ et al. Long-term effects of combined B cell immunomodulation with rituximab and belimumab in severe, refractory systemic lupus erythematosus: 2-year results. Nephrol Dial Transplant. 2020 Jun 27 gfaa117.

[2] Stohl $W$ et al. Belimumab reduces autoantibodies, normalizes low complement levels, and reduces select B cell populations in patients with SLE. Arthritis Rheum. 2012;64(7):2328-2337.

[3] Blanco et al, Age-associated distribution of B and plasma cell subsets in peripheral blood - J Allergy Clin Immunol 2018141 2208-2219.

Disclosure of Interests: Eline J. Arends: None declared, Mihaela Zlei: None declared, Christopher M. Tipton: None declared, Zgjim Osmani: None declared Sylvia Kamerling: None declared, Ton Rabelink: None declared, Ignacio Sanz: None declared, Jacques J.M. van Dongen Paid instructor for: BD Biosciences: Educational Services (fees for LUMC), Consultant of: BD Biosciences and Cytognos (fees for LUMC), Grant/research support from: GSK (flow cytometry studies for GSK BLISS-BELIEVE study NCT03312907), Cees van Kooten: None declared, Y.K. Onno Teng Consultant of: Aurinia provided financial compensation for consultancy, Grant/research support from: GSK provided belimumab for free for the Synbiose-2 clinical trial and provided an unrestricted grant to conduct the study.

DOI: 10.1136/annrheumdis-2021-eular.248

\section{POS0681 VOCLOSPORIN FOR LUPUS NEPHRITIS: INTERIM ANALYSIS OF THE AURORA 2 EXTENSION STUDY}

A. Saxena ${ }^{1}$, P. Mina-Osorio ${ }^{2}$, C. Mela ${ }^{3}$, V. Berardi ${ }^{2} .{ }^{1} N Y U$ Langone Health, Rheumatology, New York, United States of America; ${ }^{2}$ Aurinia Pharmaceuticals, Inc., Medical Affairs, Victoria, Canada; ${ }^{3}$ Aurinia Pharmaceuticals, Inc., Clinical Science, Victoria, Canada

Background: Voclosporin, a novel calcineurin inhibitor $(\mathrm{CNI})$, has been tested successfully in two pivotal trials in adult patients with lupus nephritis.

Previously reported results from the Phase 3 AURORA 1 study and the Phase 2 AURA-LV study showed that compared with mycophenolate mofetil (MMF) and low-dose steroids alone, the addition of voclosporin significantly increased the renal response rate and reduced proteinuria, as measured by urine protein creatinine ratio (UPCR), in patients with lupus nephritis (LN) at approximately one year of treatment (48 weeks in AURA-LV and 52 weeks in AURORA 1).

Objectives: Patients that completed one year of treatment in the AURORA study were eligible to enroll into the two-year, blinded, controlled extension study, AURORA 2. Here we report the first interim analysis of the ongoing AURORA 2 study.

Methods: Patients completing AURORA 1 were eligible to continue the same randomized treatment of voclosporin $(23.7 \mathrm{mg} B I D)$ or placebo, in combination with MMF (1 g BID) and low-dose oral steroids in the AURORA 2 extension. This interim analysis evaluated UPCR and estimated glomerular filtration rate (eGFR) in patients with up to two years of total treatment: one year from AURORA 1 and up to one year in AURORA 2.

Results: 116 patients in the voclosporin arm and 100 patients in the control arm enrolled in the extension study, of which 73 patients in the voclosporin arm and 51 patients in the control arm had received two years of treatment at the time of this interim analysis. Mean UPCR at pre-treatment (AURORA 1) baseline was $3.94 \mathrm{mg} / \mathrm{mg}$ in the voclosporin arm $(n=116)$ and $3.87 \mathrm{mg} / \mathrm{mg}$ in the control arm $(n=100)$. The LS mean change in UPCR from pre-treatment baseline to year two was $-3.1 \mathrm{mg} / \mathrm{mg}$ for the voclosporin arm $(\mathrm{n}=73)$ and $-2.1 \mathrm{mg} / \mathrm{mg}$ for contro arm ( $n=51$; Table 1). Mean eGFR at pre-treatment (AURORA 1) baseline was $79.6 \mathrm{~mL} / \mathrm{min}$ for the voclosporin arm $(\mathrm{n}=116)$ and $78.9 \mathrm{~mL} / \mathrm{min}$ for the contro arm $(n=100)$ and at year two, was $79.0 \mathrm{~mL} / \mathrm{min}$ for the voclosporin arm $(n=73)$ and $82.9 \mathrm{~mL} / \mathrm{min}$ for the control arm $(n=51)$. There was a small early decrease in mean eGFR in the first four weeks of treatment (in AURORA 1) after which eGFR remained stable throughout year one and year two. Additionally, there 